\title{
Furan Forming Reactions of cis-2-Alken-4-yn-1-ones
}

\author{
Charles P. Casey* and Neil A. Strotman \\ Department of Chemistry, University of Wisconsin-Madison, Madison, WI 53706 \\ casey@.chem.wisc.edu
}

Supporting information

S2. ${ }^{13} \mathrm{C}$ NMR spectrum of 1-phenyl-cis-2-penten-4-yn-1-one (cis-1)

S3. ${ }^{13} \mathrm{C}$ NMR spectrum of 1-phenyl-trans-2-penten-4-yn-1-one (trans-1)

S4. ${ }^{1} \mathrm{H}$ NMR spectrum of $c i s-1,2-\mathrm{di}(2-(5-$ phenylfuryl)ethene (cis-2) and trans-1,2-di(2-(5-phenylfuryl)ethene (trans-2)

S5. ${ }^{13} \mathrm{C}$ NMR spectrum of trans-1,2-di(2-(5-phenylfuryl)ethene (trans-2)

S6. ${ }^{13} \mathrm{C}$ NMR spectrum of 1-phenyl-5-trimethylsilyl-cis-2-penten-4-yn-1-one (cis-3)

S7. ${ }^{13} \mathrm{C}$ NMR spectrum of 1-phenyl-5-trimethylsilyl-trans-2-penten-4-yn-1-one (trans-3)

S8. ${ }^{13} \mathrm{C}$ NMR spectrum of trans, trans-1,2,3-tri(2-(5-phenylfuryl)cyclopropane (4)

S9. ${ }^{13} \mathrm{C}$ NMR spectrum of 5-Isopropylamino-1-phenyl-trans,trans-2,4-pentadien-1-one (5)

S10. ${ }^{13} \mathrm{C}$ NMR spectrum of triethyl(2-(5-phenylfuryl))ammonium chloride (7)

S11. ${ }^{13} \mathrm{C}$ NMR spectrum of 5-(2,2-dichloroethenyl)-2-phenylfuran (8)

S12. ${ }^{13} \mathrm{C}$ NMR spectrum of (dichloromethyl)triethylammonium chloride (9)

S13. ${ }^{1} \mathrm{H}$ NMR spectrum of cis-1 during reaction with DOAc 


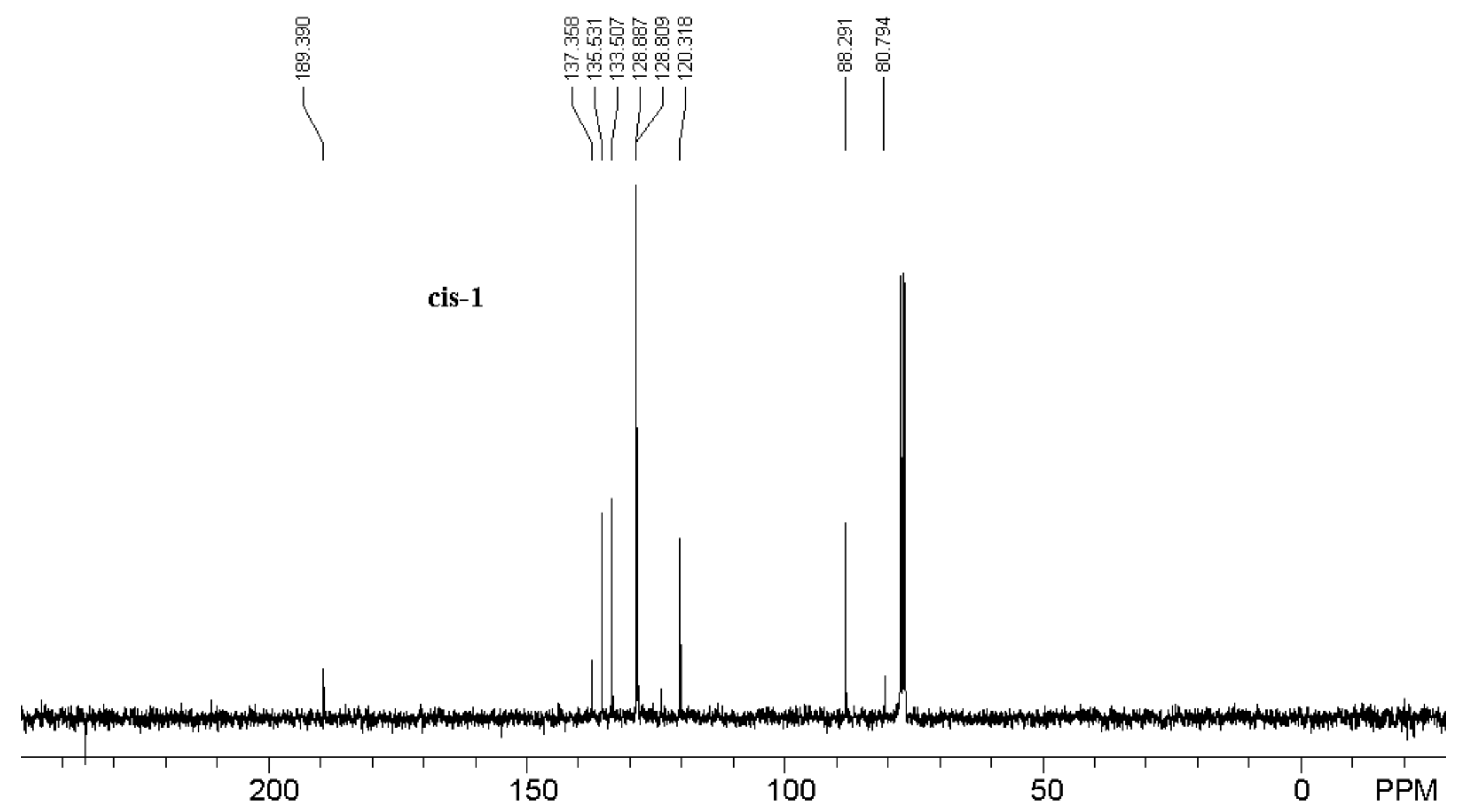




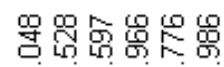

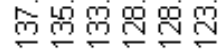

(1)

总总

少

$\left.\right|^{\infty}$

trans-1

勧

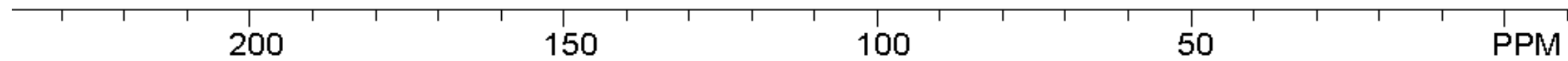




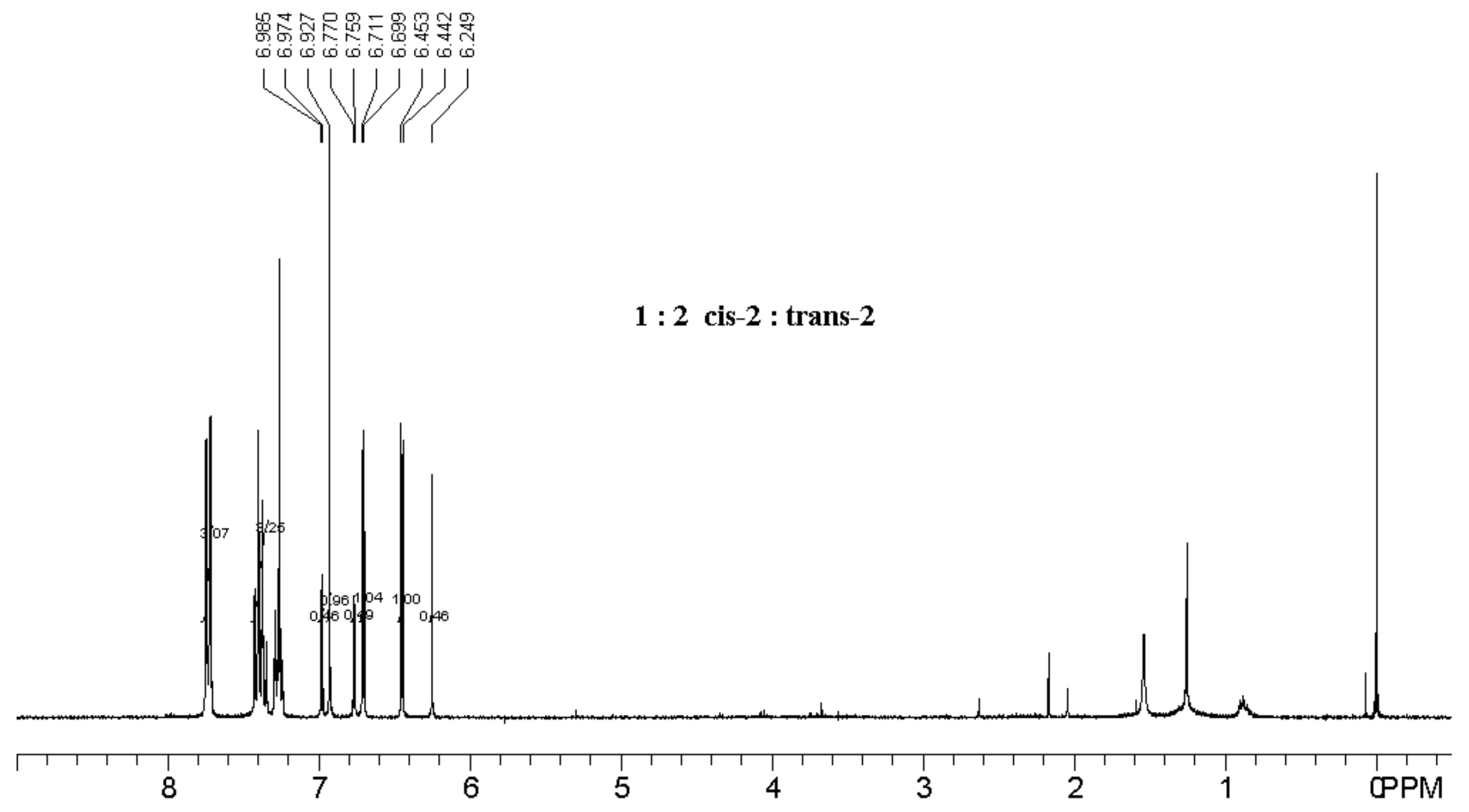




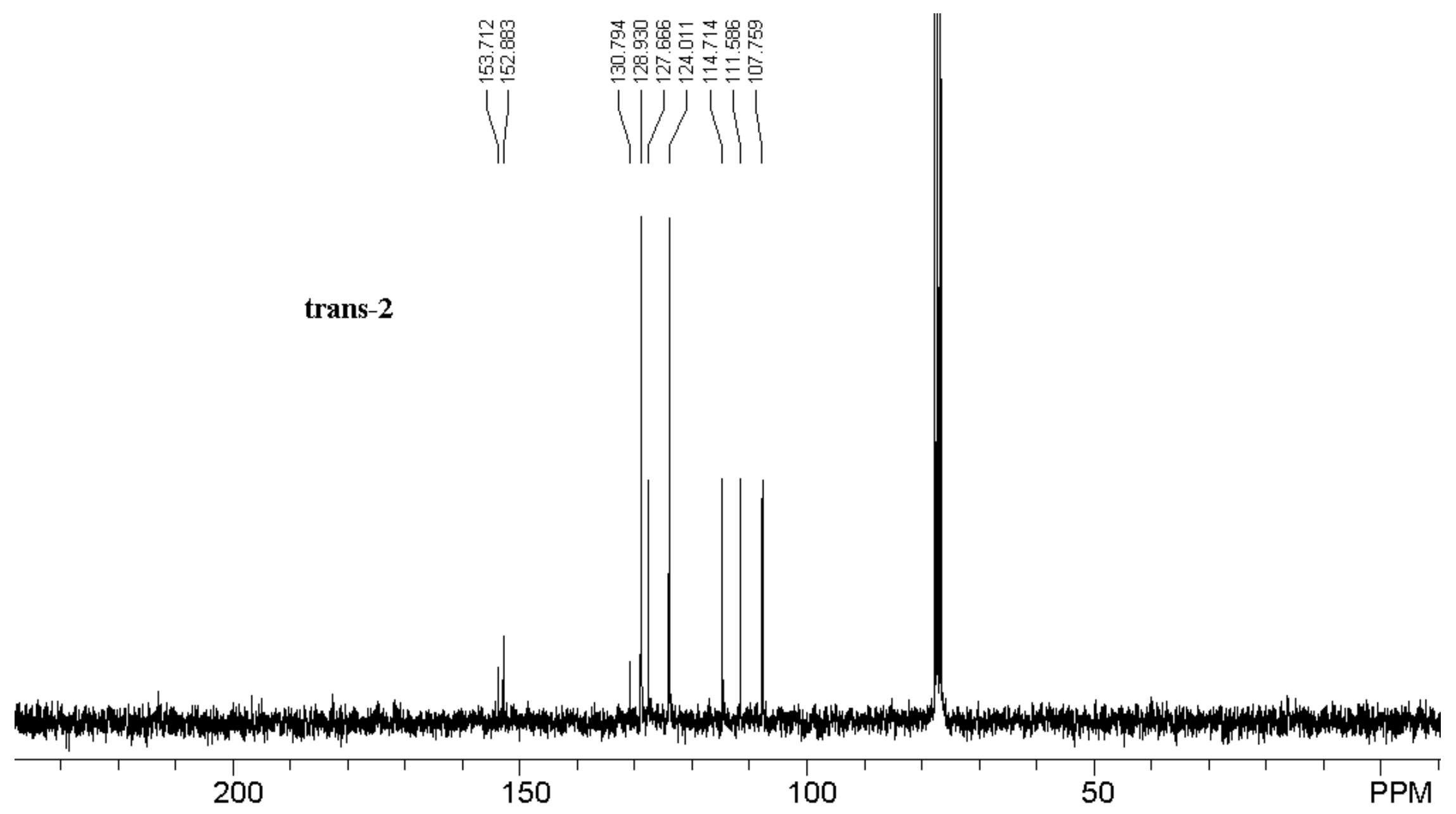




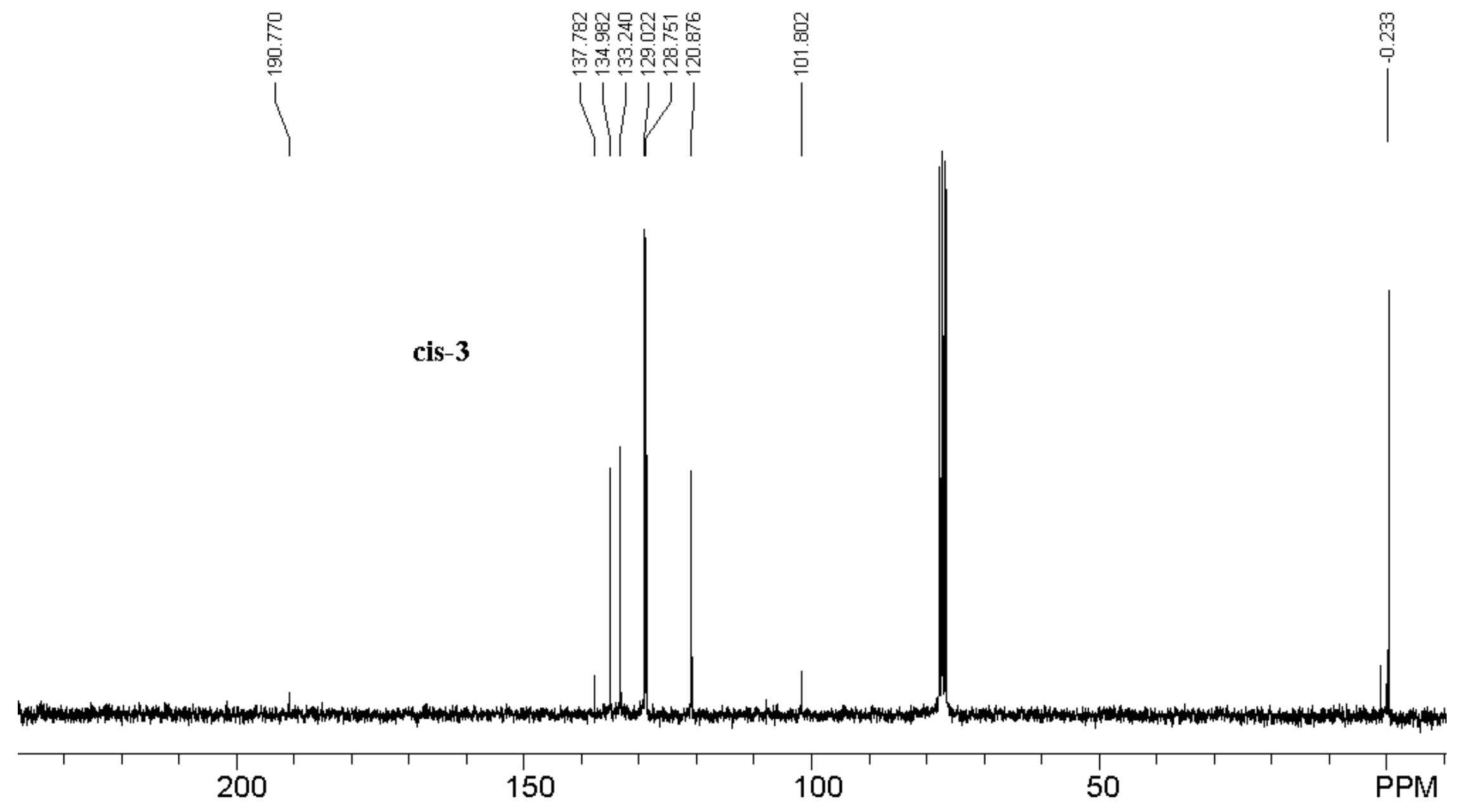



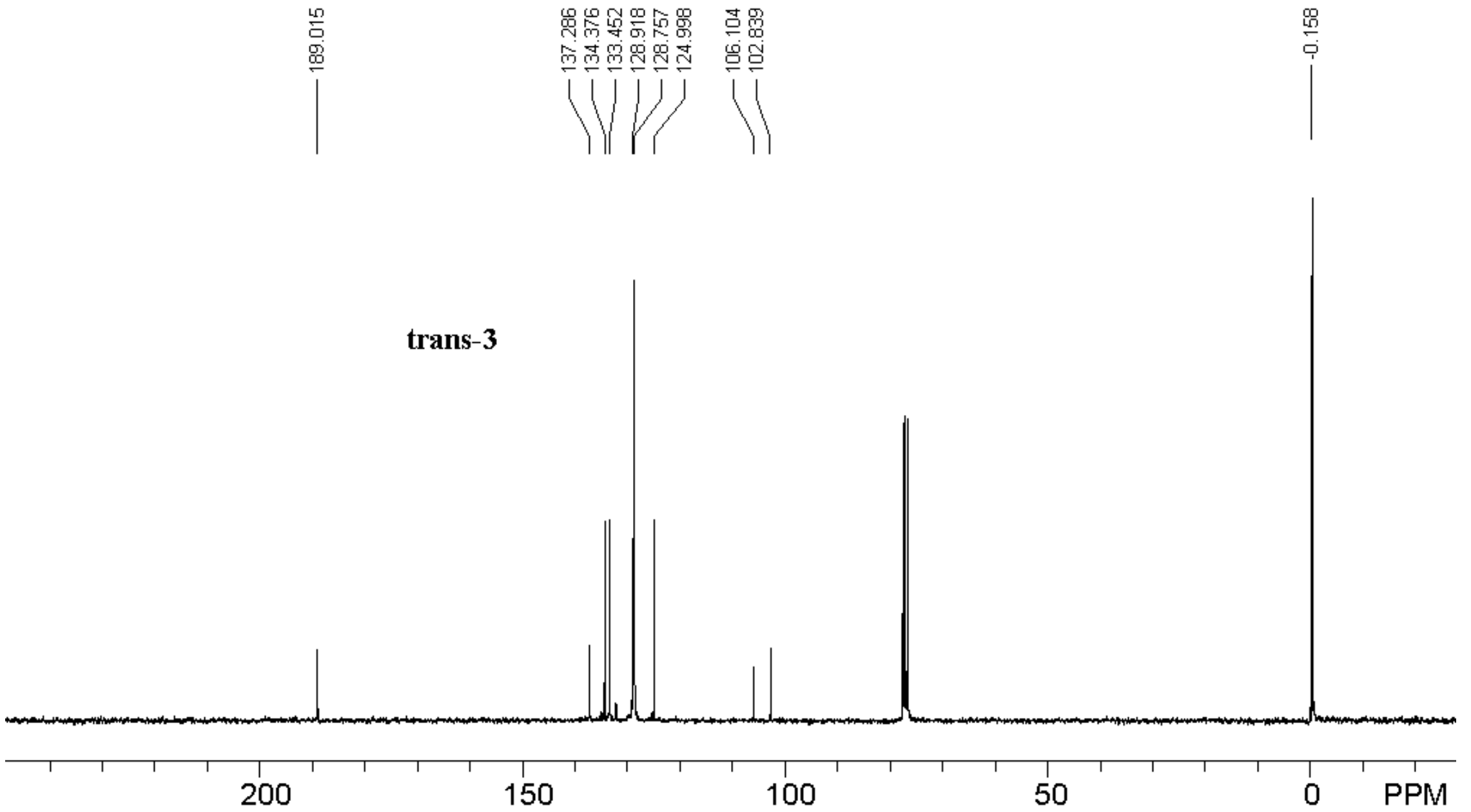


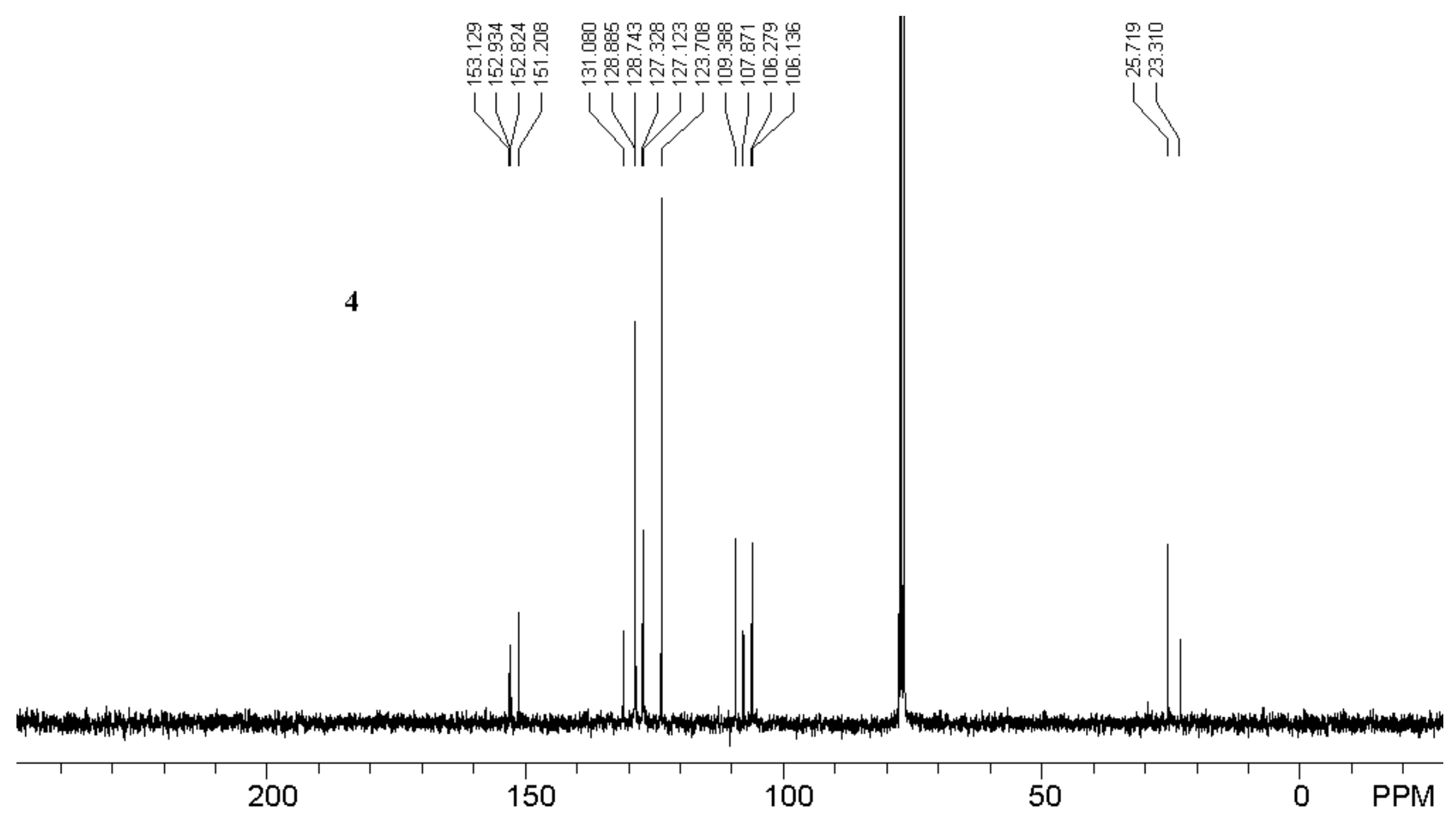




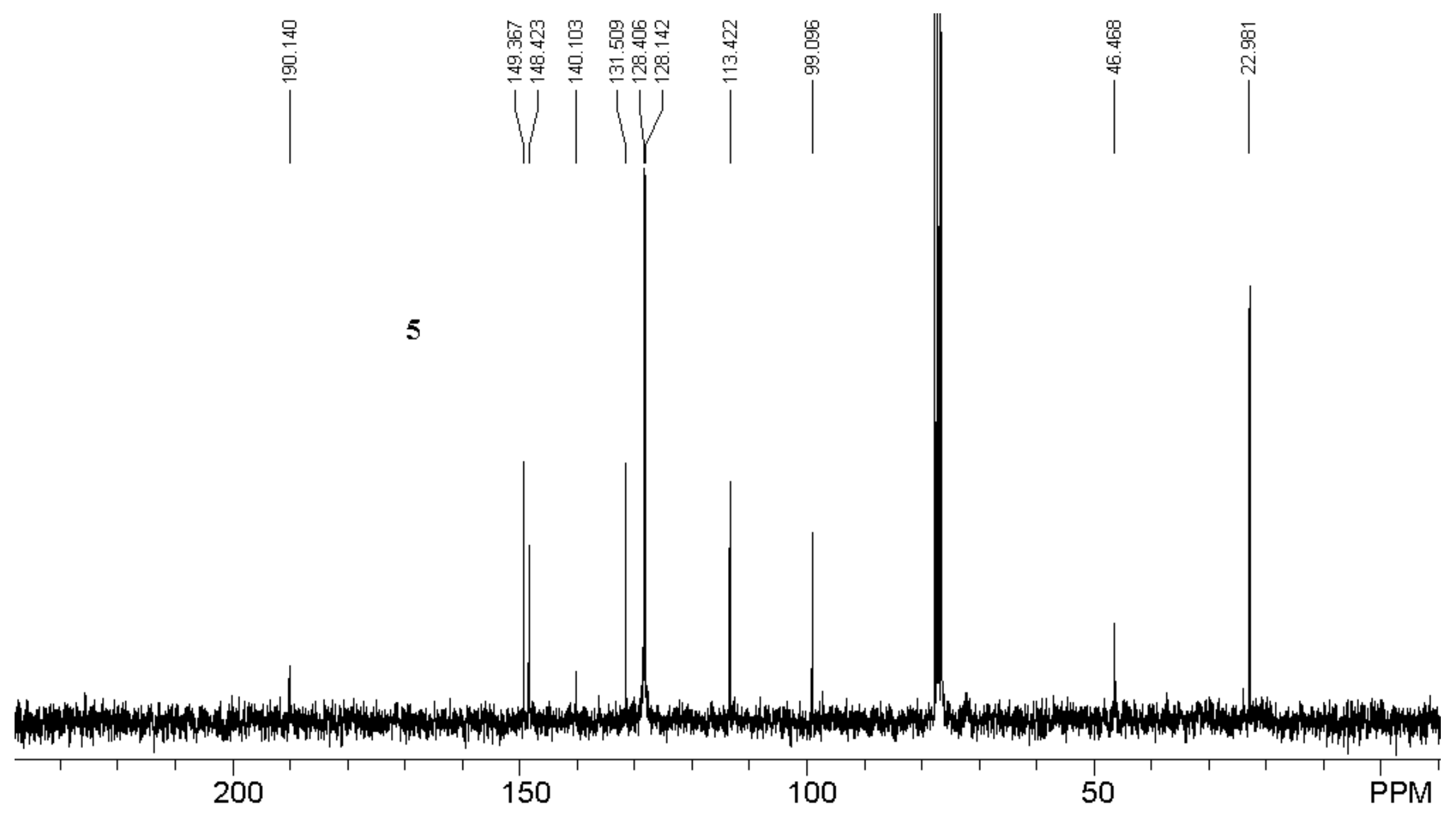




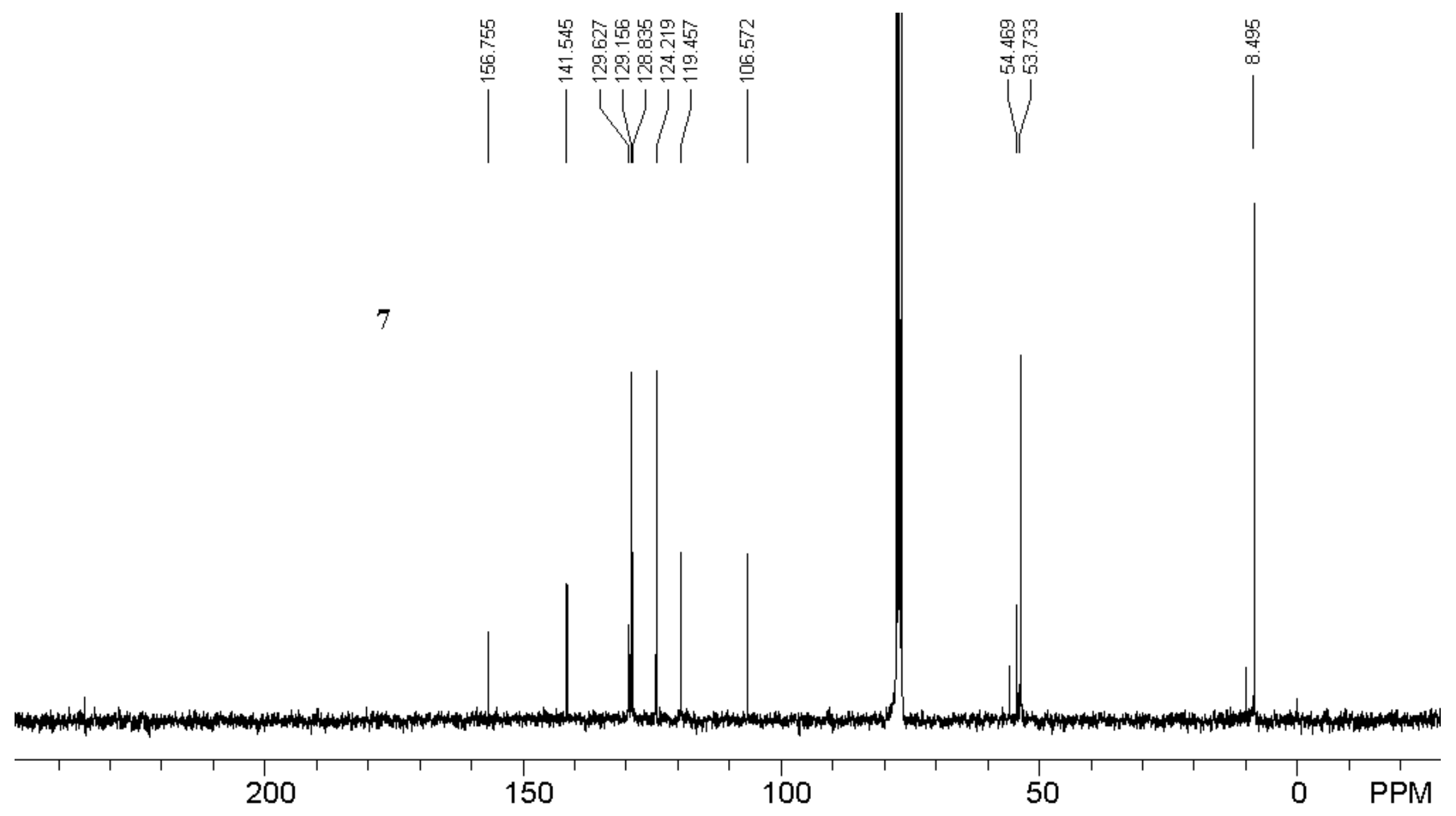




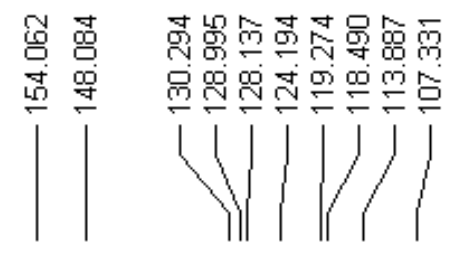

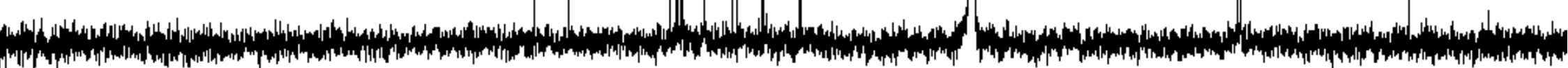

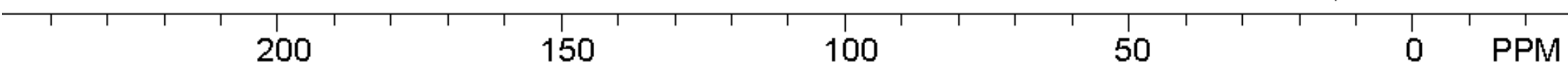




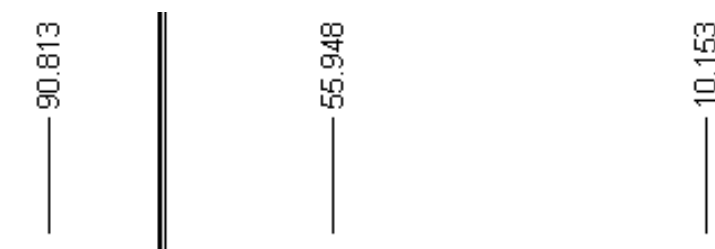

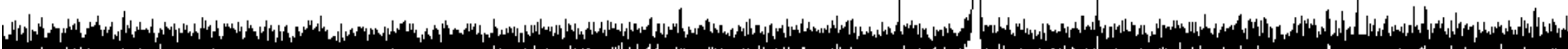



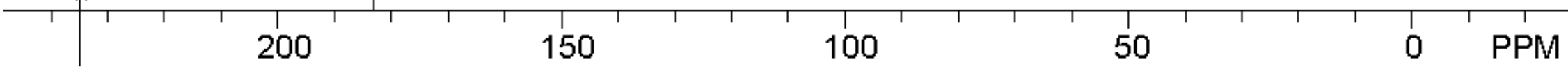


cis-1 during reaction with DOAc at $90 \%$ conversion

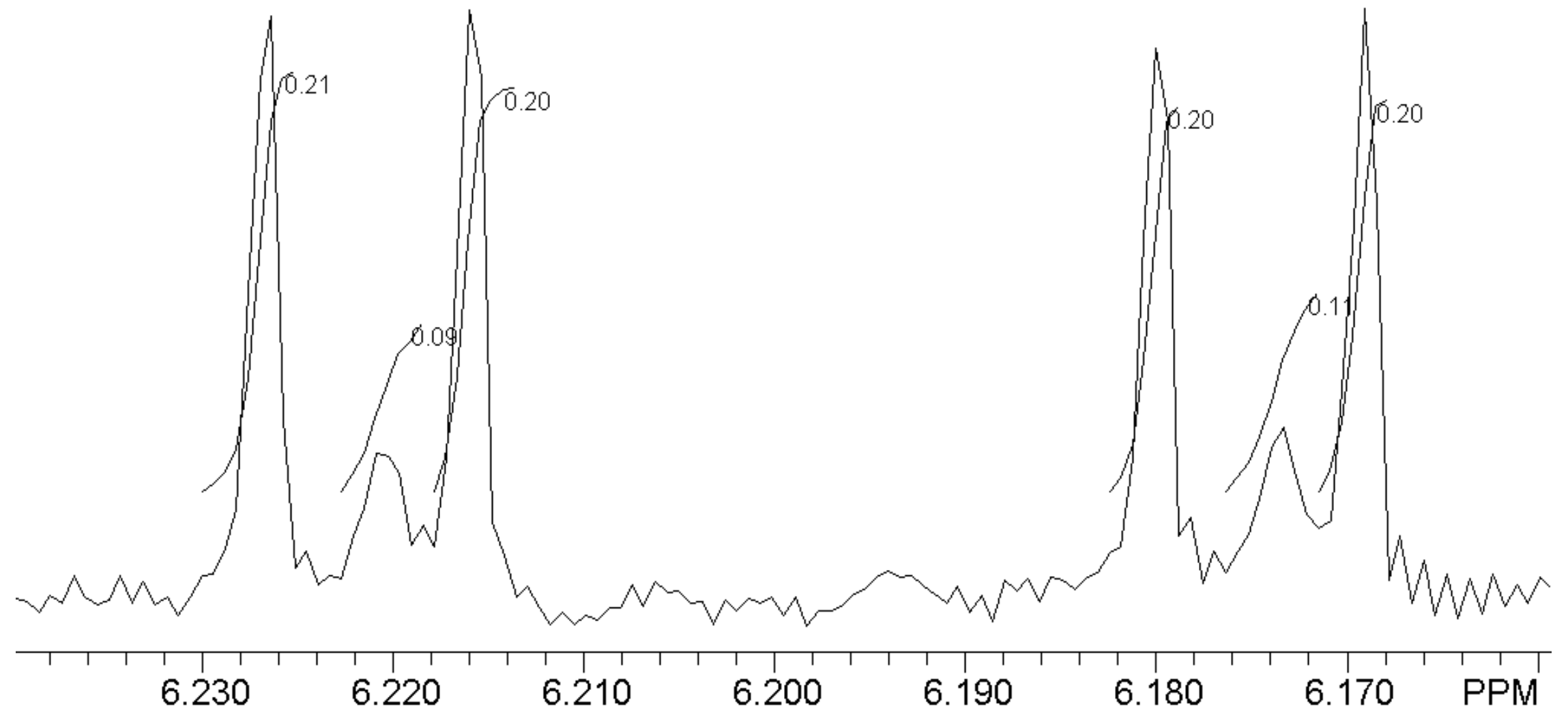

